\title{
DUST AND STAR FORMATION
}

\author{
D. Mc NALLY
}

University of London Observatory, Mill Hill Park, London NW7 2QS, England

\begin{abstract}
The view that dust is essential to star formation is challenged on the ground that other interstellar constituents can provide more rapid cooling. From the evidence of stellar minimum masses it is suggested that self absorption of the radiation emitted by the coolant $\mathbf{H}_{2}$ is the dominant mechanism leading to the heating of a collapsing fragment. It is however shown that extensive dust and molecule formation may take place during star formation and that a natural explanation for the 4 terrestrial planets in the neighbourhood of the Sun is then provided.
\end{abstract}

\section{Introduction}

The presence of interstellar dust is usually considered to be a necessary prerequisite for the initiation of star formation. The basis for this hypothesis is the observation that young stars and regions of great obscuration seem to be associated spatially. However, this observation, as was pointed out by Ambartsumian (1958) could also be interpreted on the basis that dust formed during the star formation process. It is the purpose of this paper to examine the role that is played by dust in promoting or hindering starformation. Unfortunately the role of dust will be different according to the model of star formation chosen. For example in a model of star formation based on cosmological considerations such as that proposed by Layzer (1964), dust probably plays a minor role. The same is true of the random accretion model proposed by McCrea (1960) though it is probable that the presence of dust may be important at a late stage in this model. The classical model of star formation through collapse under gravity is probably the most sensitively dependent on the assumed dust content of interstellar space and, as a result of recent numerical studies, is the model most susceptible to definitive analysis. Although the physical state of the interstellar gas is ill defined particularly in regions of high density, the numerical calculations that already exist underline those parts of the collapse where the physical state of the gas plays an important role. However, the importance of dust in this model may only relate to the opacity of the gas and in some circumstances the role of dust may be simply to provide a catalytic surface for the formation of molecular species.

\section{Models for Star Formation}

Many different models for star formation have been proposed - collapse under gravity, association of small cloudlets, cosmological condensation, ejection from galactic nuclei (see McNally, 1971). These theories have attempted to formulate a broad framework within which processes leading to star formation could operate. Most of 
the theories have concentrated on dynamical considerations to the exclusion of any considerations of whether the state of the material out of which stars would form could support the dynamics proposed. In consequence such theories of star formation, for all their apparent universality, could not give detailed models of star formation.

Recently attention has been paid to the state of the interstellar gas. The work of Hayashi and his collaborators (e.g. see Hattori et al., 1969) on the rates of likely interstellar cooling and heating processes has shown very clearly the influence that the state of the interstellar gas is likely to have. Field et al. (1969) have also shown the influence of the balance of heating and cooling mechanisms on the structure of the interstellar gas. Their work has shown clearly that unless the state of the interstellar gas is considered, it is misleading to consider the dynamics of star formation in isolation. It would be equally misleading to give undue weight to considerations based on the state of the gas alone. Unfortunately the simultaneous consideration of dynamics and the state of the interstellar material creates an intractable algebraic formulation of the equations of motion.

In one particular model of star formation - namely that of collapse under gravity some progress has been made in the simultaneous consideration of the state of the interstellar gas and its dynamics. Gravitational collapse is based simply on the result that for a given gas density and temperature (neglecting rotational and magnetic forces) there is a critical mass for an isolated gas cloud above which gravitational forces dominate all other forces - in this case gas pressure. By maintaining a situation in which gravitational forces dominate pressure forces, a gas cloud will collapse. A simple way of maintaining such collapse is by efficient cooling of the gas cloud so that the kinetic temperature and so gas pressure, remain low and gravity is allowed to dominate.

Such a simple picture has considerable appeal for gravitation is a long range associative force. But the simple model has to face formidable difficulties. Since gravity is a weak force, a gas cloud of great mass $\left(\gtrsim 1000 M_{\odot}\right)$ is required to produce collapse under 'average' interstellar conditions. The problem of forming stellar objects whose masses lie in the range $0.1-100 M_{\odot}$ must then be faced. If the interstellar gas cloud is rotating, centrifugal forces have been considered to halt collapse after the cloud density has increased by a factor of 10 (a further factor of $10^{22}$ being required before stellar densities are achieved). These problems have been considered so formidable that other models of star formation have been sought. Three dimensional calculations now being carried out by the author (unpublished) suggest that the rotational constraint is not as severe as had been expected. Nevertheless despite these difficulties, it is useful to consider collapse under gravity since it can be described effectively in mathematical terms in a form suitable for numerical solution. Several authors (Disney et al., 1969; Hunter, 1969; Larson, 1969; Penston, 1969) have tackled the problem of the collapse of gas clouds from the interstellar state for the case of spherical symmetry with no magnetic field or rotation, while Bodenheimer (1969), Hayashi (1970), and Larson (1969) have considered the collapse of more condensed objects of stellar mass to the proto-stellar state (i.e. the appearance of the object in the Hertzsprung-Russell 
diagram). While a consensus on how a spherically symmetrical gas cloud will collapse has been achieved, there is no such consensus on proto-stellar evolution and there is no real linkage of the cloud collapse problems with the evolution of proto-stellar objects. This state of affairs is not surprising. Leaving aside problems of a purely technical kind in the numerology, the physical description of all but the initial phases of any collapsing model is wholly inadequate. This is not merely a problem of available resources but the phenomena dominant throughout the range of densities and temperatures encountered during collapse are indifferently determined where they are known at all. Those workers who have tried to investigate the interaction of atomic, molecular and other processes with the dynamics of collapse have the double handicap of a difficult numerical problem and an essentially undefined physical situation. In this context wide divergences between authors are to be expected.

Nevertheless, the numerical studies have indicated an essential dynamical feature of gravitational collapse which had not been stressed sufficiently in the past. This is best demonstrated by consideration of free fall collapse (i.e. gravity is the only force acting). Motion under gravity alone takes a characteristic form. Changes in density take place slowly at first but take place at a rate which is accelerating. This means that rapid changes of density take place towards the end of collapse. In a gravitationally dominated situation collapse proceeds slowly at first and ultimately increases very rapidly. This phenomena is illustrated in Figure 1, for a slightly more realistic situation than free fall. Gas pressure and energy loss can be considered in a system in which the gas pressure is not zero provided the velocity distribution is assumed to be linear wave (i.e. the velocity increases linearly from centre to surface of the collapsing cloud).

In linear wave flow the initial density distribution is maintained so that $\rho / \rho_{i}$ refers to any point in the gas cloud so long as the same relative position at all times is considered. The model used to determine Figure 1. is one in which a linear wave flow collapse from equilibrium is initiated either by a sudden initial acceleration or cooling and which attains a final radiating equilibrium state. From Figure 1 , it is clear that $75 \%$ of the collapse time must elapse before the density increases by an order of magnitude. However, an increase in density by three orders of magnitude take place in the next $15 \%$ of the collapse time and a very rapid increase of density takes place in the very last stage before the final equilibrium configuration is reached. Such characteristic behaviour has an important consequence. Disney et al. (1969) investigated the collapse of a gas cloud when cooled by some physically realistic process (within wide limits it does not matter what the precise process is). They found that in the denser hotter central part of the cloud cooling was rapid while it was slow in the cooler less dense region near the boundary. As a consequence collapse under gravity was scarcely resisted by gas pressure in the cloud centre whereas collapse in the outer parts of the cloud proceeded rather slowly because gas pressure and gravitational forces remained of similar order. Such a situation capitalised on the characteristic nature of gravitational collapse to produce a region of high density near the cloud centre while the bulk of the gas cloud did not vary in density by a significant factor from the initial density. Such a situation gives separation of a collapsing region of small mass from a more 


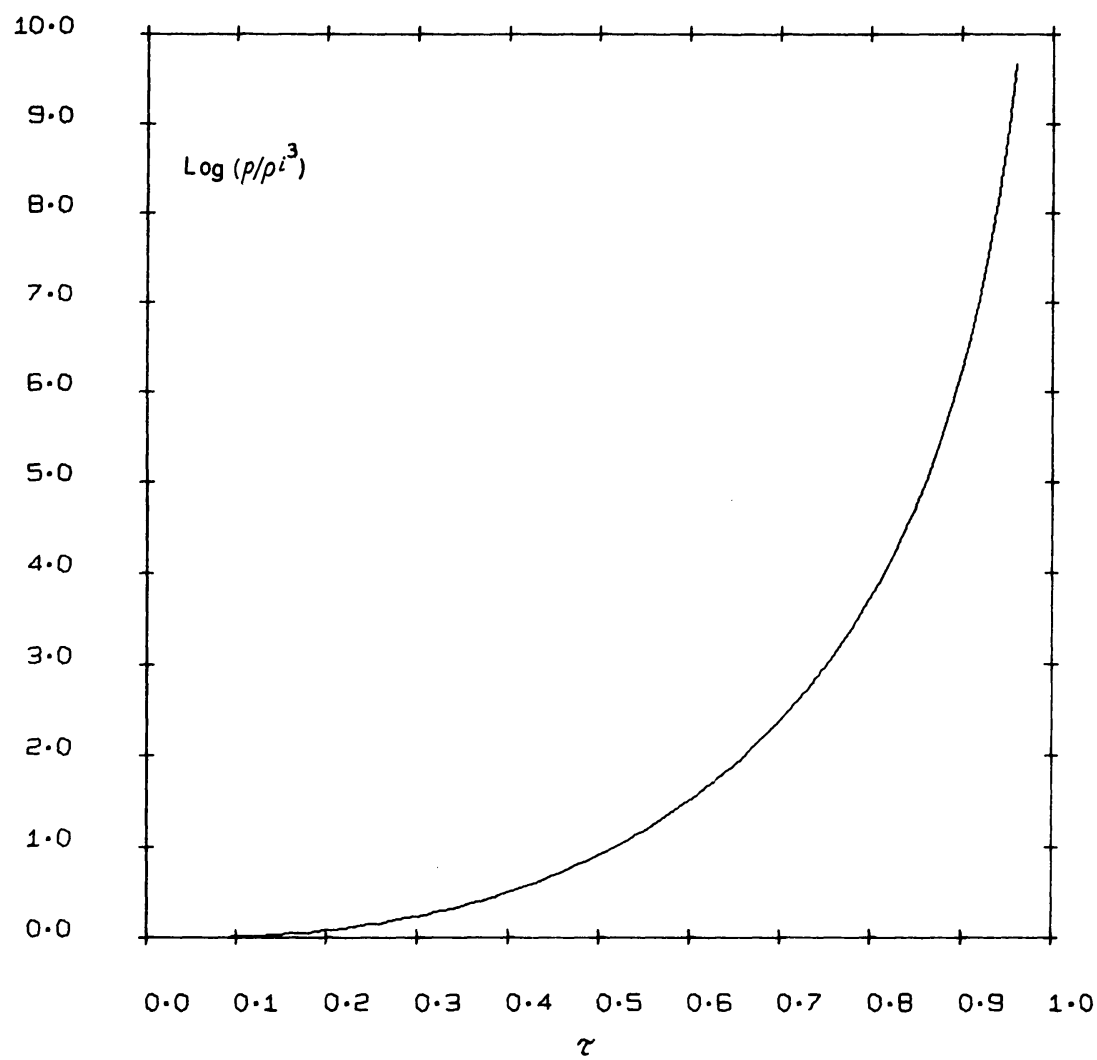

Fig. 1. The variation of density ( $\rho-$ with respect to an initial density $\left.\rho_{l}\right)$ with time $(\tau-$ relative to the collapse time for the system considered) in the case of a gas cloud collapsing with linear wave flow.

massive parent cloud. However, in the context of the present paper it is the possibility of the existence of a linking region of great density and temperature variation connecting the high density and hot collapsing fragment and the low density cool surrounding gas that is of importance. If the collapsing fragment is attaining stellar densities a density variation more than $10^{20}$ may be expected in the linking region accompanied by a temperature variation by at least a factor of $10^{3}$. If such a region exists surrounding a collapsing fragment the consequences for molecule and dust formation may be considerable.

Disney et al. (1969) also drew attention to the possibility that opacity in the interstellar medium may well inhibit the rate at which heat can be removed from the interstellar gas. Opacity is contributed by the general opacity of the dust component and self-absorption by the coolants themselves. They showed that opacity effects would not become apparent until the stage of rapid density increase was attained. They therefore assumed a sudden transition from a cooling to an adiabatic regime in the collapsing fragment. More detailed calculations showed that the rate of the transition was unimportant since the rate of density increase was rapid. They found for adiabatic 
collapse that the kinetic temperature of the gas increased and that eventually pressure forces would exceed the gravitational. In such a situation re-expansion would occur. To maintain collapse they proposed that the ionisation of atomic hydrogen would be a sink for the heat of compression. However, for a given value of the opacity a minimum collapsing mass was required in order to raise the temperature of the gas to a value where collisional ionisation was important. It is of some interest that the value of this mass depended both on the opacity of the interstellar gas and the properties of atomic hydrogen. The limiting mass $M_{c}$ is given by

$$
M_{c}=1.4 \times 10^{29} \alpha T_{i} T_{c},
$$

where $\alpha$ is related to the opacity of the gas, $T_{i}$ is the temperature of the gas before the effect of opacity operates and $T_{c}$ is the temperature at which pressure and gravitational forces balance. Clearly for small values of $M_{c}$, small values of $\alpha, T_{i}$ and $T_{c}$ are required. In general $T_{i}$ can be maintained at about $10 \mathrm{~K}$ and $T_{c}$ is fixed by the temperature at which hydrogen becomes collisionally ionised $\left(\sim 10^{4} \mathrm{~K}\right)$. The value of $\alpha$ is therefore critical. If $\alpha$ is locally large then stars of large mass would be expected whereas if $\alpha$ were locally small then stars of low mass would be expected. A similar argument applies if dissociation of $\mathrm{H}_{2}$ is used as the sink of compressional heating. It is essential therefore to know what component of the interstellar medium contributes most to $\alpha$ at the time when radiative cooling becomes inefficient in removing compressional heating.

Two properties of the interstellar gas are therefore important at two stages in the star formation process namely the cooling of the interstellar gas to initiate and maintain the early collapse stage and the opacity of the interstellar gas in retarding that collapse. Interstellar dust clearly contributes both to the cooling of the interstellar gas and to its opacity. But is the dust the dominating component of the interstellar medium? The contributions to cooling and opacity of the interstellar gas will be considered in the next two sections.

\section{The Cooling of the Interstellar Gas}

The interstellar gas can be cooled by a number of its constituents. The contribution to the cooling of $\mathrm{O}, \mathrm{H}_{2}$, atomic ions (in particular $\mathrm{C}^{+}, \mathrm{Si}^{+}, \mathrm{Fe}^{+}, \mathrm{Mg}^{+}$) and dust are compared in Figure 2. It is clear from an inspection of Figure 2 that there are several ways in which the interstellar gas could rid itself of energy through radiative processes. It might be argued that interstellar $\mathrm{O}, \mathrm{C}, \mathrm{Fe}, \mathrm{Si}, \mathrm{Mg}$ could be depleted by being components of the dust. This would still leave $\mathrm{H}_{2}$ as a more effective coolant than the dust. Dust is the least efficient of the proposed coolants by several orders of magnitude so that almost total depletion of the interstellar gas of all atomic species other than hydrogen, in addition to impossibility of formation of molecular hydrogen, would be required before dust could be considered as an effective coolant. Therefore interstellar dust cannot be considered to play a dominant role as a coolant and therefore plays little part in initiating collapse through differential cooling of the interstellar gas. 


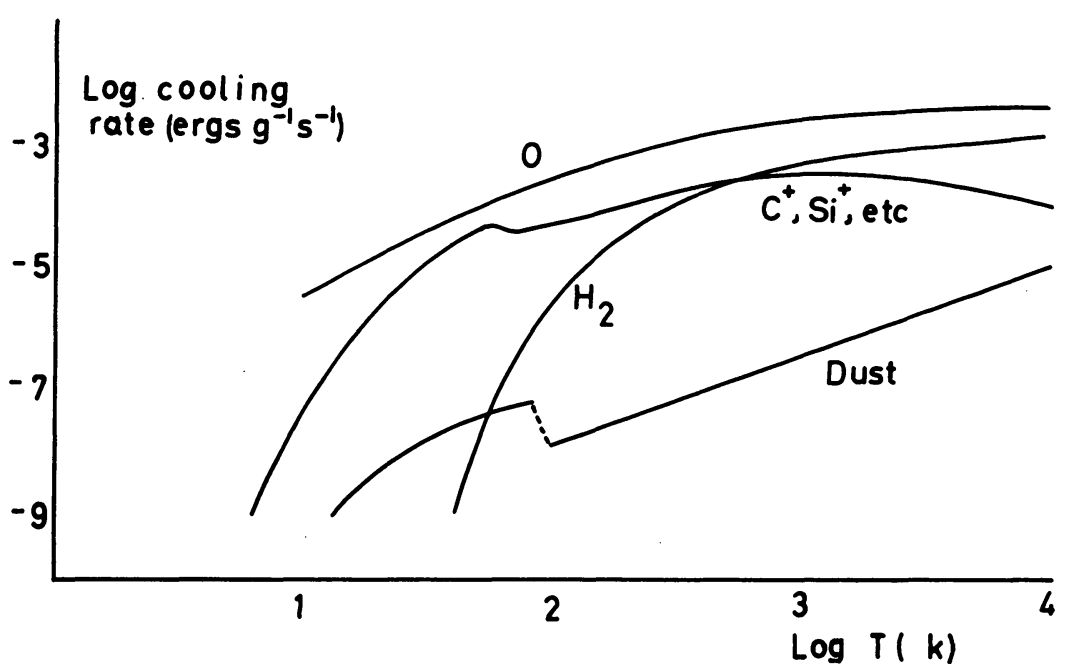

Fig. 2. A comparison of interstellar cooling rates (adapted from Disney et al., 1969).

\section{Opacity of the Interstellar Gas}

The opacity of the interstellar gas is conventially considered in terms of the amount of extinction caused by the dust. While the dust will contribute to the absorption of the radiation emitted by coolants, the self-absorption of this radiation by the coolants must also be considered. Such self-absorption plays an important part when considering opacity at the wavelength of the cooling radiation (in the infra-red).

Hattori et al. (1969) have considered in some detail the cross sections for self-absorption by some of the interstellar coolants considered in the last section. The absorption cross section for dust has been adapted from the study of interstellar opacity by Gaustad (1963). For the purpose of illustration the quantity $\alpha$ of Equation (1) has been evaluated from the cross section $\sigma(a)$ for the species $a$.

$$
\alpha^{-1}=\varrho l(a)=M_{\mathrm{H}} n(\mathrm{H}) l(a),
$$

where $\rho$ is the gas density, $M_{\mathrm{H}}$ is the mass of a hydrogen atom, $n(\mathrm{H})$ is the number density of $\mathrm{H}$ atoms and $l(a)$ is a characteristic length defined by:

$$
l(a)=\frac{1}{n(a) \sigma(a)}=\frac{1}{n(\mathrm{H}) A(a) \sigma(a)},
$$

where $A(a)$ is the abundance of species $a$. From Equations (2) and (3)

$$
\alpha=A(a) \sigma(a) / M_{\mathrm{H}} .
$$

Values of $\alpha$ for $\mathrm{C}^{+}, \mathrm{S}^{+}, \mathrm{O}, \mathrm{H}_{2}$ and dust are given in Table $\mathrm{I}$.

It is clear from Table $\mathrm{I}$ that radiation from $\mathrm{H}_{2}$ is least readily absorbed while that from $\mathrm{O}$ is most easily self-absorbed. This means that at an early stage in the collapse $\mathrm{O}, \mathrm{C}^{+}$, and $\mathrm{Si}^{+}$cease to be effective coolants. Only $\mathrm{H}_{2}$ remains free of self-absorption 
TABLE I

The relative opacity of interstellar constituents evaluated at $100 \mathrm{~K}$

\begin{tabular}{llll}
\hline$a$ & $A(a)$ & $\sigma(a)^{*}\left(\mathrm{~cm}^{2}\right)$ & $\alpha$ \\
\hline $\mathrm{C}^{+}$ & $3 \times 10^{-4}$ & $1.1 \times 10^{-17}$ & $2.0 \times 10^{3}$ \\
$\mathrm{Si}^{+}$ & $3 \times 10^{-5}$ & $1.7 \times 10^{-17}$ & $3.1 \times 10^{2}$ \\
$\mathrm{O}^{-18}$ & $7 \times 10^{-3}$ & $8.8 \times 10^{-18}$ & $3.7 \times 10^{4}$ \\
$\mathrm{H}_{2}$ & $10^{-1}-10^{-3}$ & $6.6 \times 10^{-25}$ & $4.0 \times\left(10^{-2}-10^{-4}\right)$ \\
dust & $3 \times 10^{-13}$ & $2 \times 10^{-11}$ & 3.6 \\
\hline
\end{tabular}

* varies at $T^{-\frac{1}{2}}$ where $T$ is the temperature

until high densities are attained. If dust can absorb the $28 \mu$ radiation from $\mathrm{H}_{2}$ ( $J=2 \rightarrow 0$ rotational transition) then dust could play the dominating role in interstellar opacity. On the basis of current concepts of the size distribution of interstellar dust particles it is unlikely that dust will be an efficient absorber of the $28 \mu$ radiation of $\mathrm{H}_{2}$. Consequently it would appear that the opacity which should be used in Equation (1) is that for self-absorption by $\mathrm{H}_{2}$. Taking a value of $10^{-2}$ for $\alpha$ and setting $\mathrm{T}_{i}=10 \mathrm{~K}$, $T_{c}=10^{4} \mathrm{~K}$ a minimum stellar mass of $1.4 \times 10^{32} \mathrm{~g}$ is predicted. Since the minimum stellar mass is of this order it is tempting to propose that it is the self-absorption of $\mathrm{H}_{2}$ which plays a dominating role in determining the minimum stellar mass. If an interstellar situation could be envisaged where neither dust nor $\mathrm{H}_{2}$ was present, then only stars of large mass would be able to form assuming the presence of the necessary coolants.

The above suggestions require detailed elaboration in a model which takes account of the cooling mechanisms available and the sources of interstellar opacity. Such a model requires a detailed solution of the equation of radiative transfer within the collapsing cloud and so far this has not been attempted. The full number of physical processes contributing to the interstellar cooling, heating and opacity may not have been fully enumerated and the detailed nature of the interstellar grains are not known. Consequently any calculation based on a partial description of the state of the interstellar gas can only give an indication of a possible path of collapse.

\section{The Formation of Dust}

If dust does not play a significant role in cooling the interstellar gas or contribute to the opacity of the gas with respect to limitation of cooling rate it may legitimately be asked - of what use is dust in the star formation process? It is clear that $\mathrm{H}_{2}$ is the most useful coolant for the interstellar gas and that it is self-absorption by $\mathrm{H}_{2}$ which effectively limits its use as a coolant. A short answer would be that dust is at best of very secondary importance to star formation. However, since a detailed model of star formation is not available such a statement must be tempered with caution - the more particularly as it seems that $\mathrm{H}_{2}$ is most efficiently produced by reactions at grain surfaces (McNally, 1972).

The role of dust may therefore lie in the production of $\mathrm{H}_{2}$ and other molecules. 
If this is the case it poses the awkward question of why the production of a simple diatomic molecule should require the presence of multiatomic dust particles. However, star formation at the present epoch may well be accompanied by formation of dust and molecular species.

The region linking a collapsing fragment with the remainder of the parent cloud is one which has a great variation of density and a smaller but still significant variation of temperature. Density variation by up to a factor of $10^{20}$ and a temperature variation by a factor of $10^{3}$ could be anticipated. At the densities and temperatures $(10 \mathrm{H}$ atoms $\mathrm{cm}^{-3}, 100 \mathrm{~K}$ ) conventionally assumed for interstellar gas clouds reaction mechanisms are slow. However, the greater the gas density the faster the reaction rates. In Figure 3 is compared the variation of the rate (see McNally, 1972) of the reaction

$$
\mathrm{C}+\mathrm{H} \rightarrow \mathrm{CH}
$$

at a grain surface with the free fall times at the same density. For densities lower than $5 \times 10^{11} \mathrm{H}$ atoms $\mathrm{cm}^{-3}$ the chemical reaction is slower than the dynamical time scale. For higher densities the rate of the chemical reaction will be more rapid than the dynamical time scale. The example chosen here is of no significance being merely used to illustrate the point that at high densities chemical reaction times will become shorter than dynamical time scales. Consequently at some stage in the evolution of a collapsing gas cloud to the stellar state there will be a situation in the region surrounding the collapsing fragment when chemical reactions will become so fast that a great variety of reactions will be able to proceed to completion and further reactions of greater complexity initiated. The type of molecule produced will depend on the conditions of density and temperature but certainly complex molecular species

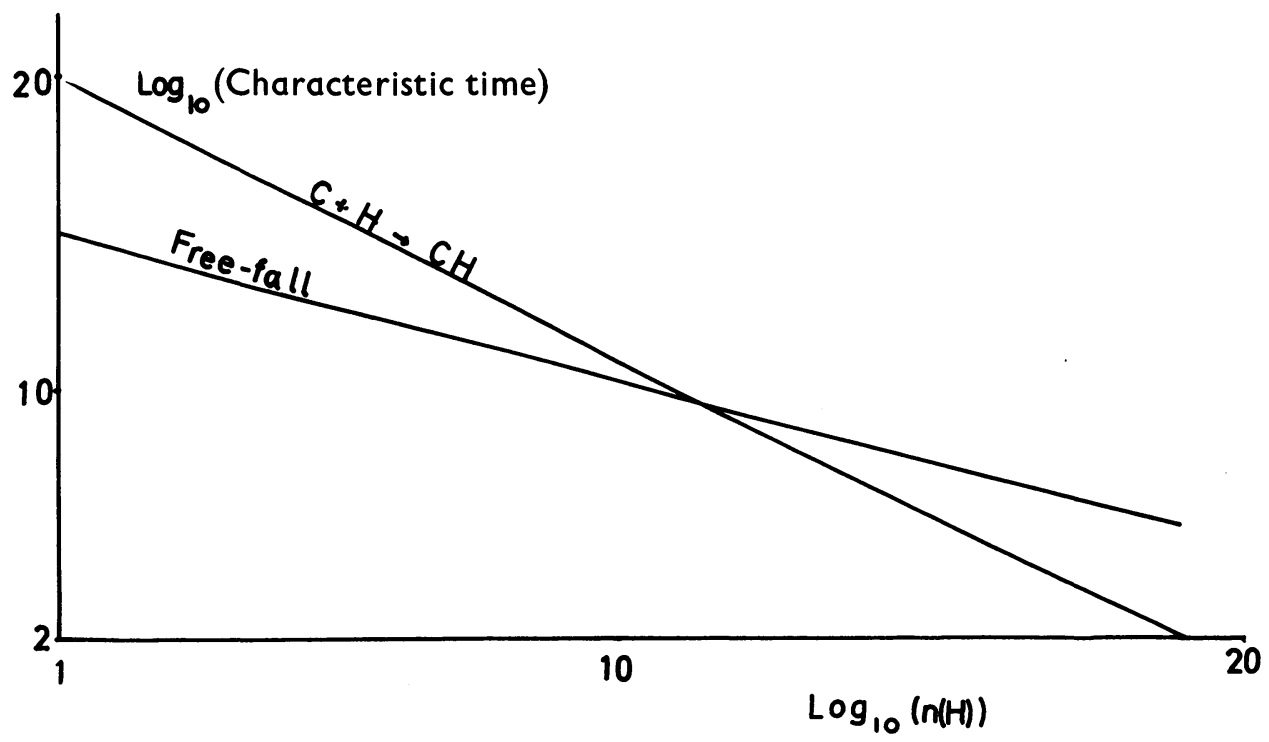

Fig. 3. A comparison of dynamical (free fall) and chemical $(\mathrm{C}+\mathbf{H} \rightarrow \mathrm{CH})$ time scales. 
and the growth and production of dust particles could be envisaged. If some initial ionisation of the linking region can decay subsequently a further range of reactions could be initiated. The chemical processes are likely to be very complex, but their rapidity will greatly alter the composition of the region linking the collapsing fragment and the remainder of the parent cloud.

On the basis of the relative opacities in Table I it would seem more plausible that extensive dust and star formation would be associated with stars of small mass. If the opacity is dominated by the self-absorption characteristics of $\mathrm{H}_{2}$, the collapse of the central core will proceed to higher densities before the second phase of star formation takes place, initiated either by the ionisation of $\mathrm{H}$ or the dissociation of $\mathrm{H}_{2}$. The existence of the terrestrial planets close to the Sun could be cited as evidence for dust and molecule production in the denser part of the linking region surrounding the collapsing fragment. However, detailed calculations are required to lift this proposal from the realm of speculation. Herbig (1970) has suggested that it is the particulate ejecta from planetary systems that maintains the interstellar dust. The recent radio and infra-red observations (see Mezger, 1971) of objects considered to be proto-stellar show evidence for both molecule and dust formation.

\section{Conclusion}

It has been shown that for star formation by collapse under gravity, interstellar dust is not likely to play a major role. The molecule $\mathrm{H}_{2}$ has been shown to play a much more important role than dust. Ambartsumian's interpretation of the observation that dust and young stars are found in close association as showing that star formation and production of dust take place at the same time is given support. Furthermore it has been argued that extensive molecule formation must also be associated with the formation of stars particularly stars of low mass. The formation of the solid terrestrial planets in the neighbourhood of the Sun therefore receives a natural explanation in that it just in the region nearest the forming Sun that dust and molecule production were most rapid.

The conventional view that dust is essential to star formation finds little support from the argument presented here. But it must be remembered that until more is known of interstellar dust, some of the numerical values used here could be considerably upset.

\section{References}

Ambartsumian, V. A.: 1958, in R. Stoop (ed.), La structure et l'évolution de l'universe, Brussels Institut International de Physique Solvay, p. 241.

Bodenheimer, P.: 1968, Astrophys. J. 153, 483.

Disney, M. J., McNally, D., and Wright, A. E.: 1969, Monthly Notices Roy. Astron. Soc. $146,123$.

Field, G. B., Goldsmith, D. W. and Habing, H. J.: 1969, Astrophys. J. 155, L149.

Gaustad, J. E.: 1963, Astrophys. J. 138, 1050.

Hayashi, C.: 1970, Mem. Soc. Roy. Sci. Liège 19, 127.

Hattori, T., Nakano, T., and Hayashi, C.: 1969, Prog. Theor. Phys. Japan 42, 781. 
Herbig, G. H.: 1970, Mem. Soc. Roy. Soc. Liège 19, 13.

Hunter, J. H. Jr.: 1969, Monthly Notices Roy. Astron. Soc. 142, 473.

Larson, R. B.: 1969, Monthly Notices Roy. Astron. Soc. 145, 271.

Layzer, D.: 1964, Ann. Rev. Astron. Astrophys. 2, 341.

McCrea, W. H.: 1960, Proc. Roy. Soc. A256, 245.

McNally, D.: 1971, Rep. Prog. Phys. 34, 71.

McNally, D.: 1972, Adv. At. Mol. Phys. 8, 1.

Mezger, P. M.: 1971, in C. de Jager (ed.), Highlights of Astronomy, Vol. 2, D. Reidel Publishing Co., Dordrecht, p. 366.

Penston, M. V.: 1969, Monthly Notices Roy. Astron. Soc. 145, 457. 